\title{
Optically controlled terahertz filter based on graphene and cross-like metasurface
}

\author{
A. N. Grebenchukov, A. D. Zaitsev, M. K. Khodzitskiy \\ ITMO University, Kronverkskiy 49, St. Petersburg, 197101, Russia \\ grebenchukov_a@mail.ru, anleza@ya.ru, khodzitskiy@yandex.ru
}

PACS 84.30.Vn, 81.05.ue, 81.05.Xj

DOI 10.17586/2220-8054-2017-8-3-342-346

\begin{abstract}
We propose a theoretical model for the optically controlled terahertz filter based on hybrid graphene/metasurface structure. Such a device has a high accuracy for the frequency adjustment by the different intensities of the optical pumping in the infrared spectral range, fast response time and polarization independence. The tuning by optical pumping of the spectral characteristics of the filter in term of resonant frequency and Q-factor was shown.
\end{abstract}

Keywords: terahertz filter, graphene, metamaterial.

Received: 20 March 2017

Revised: 12 April 2017

\section{Introduction}

Currently, devices that can generate, control and receive the radiation in the terahertz (THz) frequency range have been widely spread since the first sources and receivers of such radiation were created. Radiation in this frequency range is nonionizing in nature and easily passes through the majority of dielectrics, but is strongly absorbed by conductors and some dielectrics. These properties allow the use THz radiation in spectroscopy [1], near-field microscopy [2], scanning security systems, and also in medicine (for example, in the tomography which can visualize layers of the body up to several $\mathrm{cm}$ in depth) and in communications systems. A huge incentive for the creation of devices to control $\mathrm{THz}$ radiation is that doing so would allow the application of this radiation in many directions, including the field of the THz communications [3].

Graphene is a promising material for use as the basis of $\mathrm{THz}$ devices. Graphene is a two-dimensional allotropic modification of carbon in which its atoms are arranged in a planar hexagonal lattice. Due to its exceptional electronic and optical properties and high resistance to deformation [4], graphene may become a key component in solar cells, light emitting diodes, flexible touch screens, ultrafast lasers and frequency converters [5]. The frequency of graphene plasma waves lies in the terahertz range [6], making graphene appealing for controllable terahertz devices such as modulators and filters, where the resonant frequency can be tuned by an external electric field or optical pumping. In recent research, various hybrid structures based on graphene/metamaterial were proposed, and their optical parameters were controlled by application of a bias voltage between metamaterial and graphene [7-11] or by optical pumping in the infrared frequency range [12]. The central resonant frequency for such devices depended on the geometrical parameters of unit cell and the conductivity of graphene [8].

In this article, we propose and demonstrate an optically tunable $\mathrm{THz}$ filter based on hybrid system consisting of graphene and a metasurface with cross-like elements that provides polarization invariance for the structure. This geometry is commonly used because the resonant frequency of the structure is uniquely defined by only few parameters of the unit cell. Additionally, it is also relatively simple to manufacture such a structure. This filter has a very high accuracy for frequency adjustment by varying the intensity of the infrared radiation, and it also has a fast response time. Control via optical pumping instead of the electric field allows one to avoid such difficulties as the coating of the composite structure by the microelectrodes and usage of the electronic circuits to control the characteristics of the filter. In accordance with these advantages, such a device may find application in $\mathrm{THz}$ communications systems.

\section{Optical pumping of graphene}

The surface conductivity model is used to describe the properties of graphene as its numerical value can be measured across a wide frequency range [13].

The gapless band structure of graphene leads to unusual behavior for its conductivity. So, for the terahertz frequency range, the dependence of graphene's conductivity upon the frequency of the incident radiation is observed. Under the influence of optical pumping in the visible and infrared frequency range, the photogeneration of 
electron-hole pairs with an efficiency of $2.3 \%$ is observed. Immediately after pumping the electron-hole pairs are tend to transformed into the lower-energy state.

Optical generation of the electron-hole pairs in graphene is described by the quasi-Fermi level $\mu$ (the chemical potential), which for the nonexcited state of graphene, is located at the intersection of the valence band and conduction band, and its energy is equal to zero.

The theoretical value of the surface conductivity for graphene can be calculated using the Kubo formula [14], which gives the expressions for the complex conductivity for the graphene monolayer, which takes into account both the intraband $\hat{\sigma}_{\text {intra }}$ and the interband $\hat{\sigma}_{\text {inter }}$ transitions, depending on the frequency $\omega$ :

$$
\begin{gathered}
\hat{\sigma}_{\text {intra }}(\omega)=\frac{2 e^{2} k_{B} T \tau}{\pi \hbar^{2}(1-i \omega \tau)}\left[\ln \left(\exp \left(\frac{\mu}{k_{B} T}\right)+1\right)-\frac{\mu}{2 k_{B} T}\right], \\
\hat{\sigma}_{\text {inter }}(\omega)=\frac{e^{2}}{4 \hbar}\left[\frac{1}{2}+\frac{1}{\pi} \operatorname{atan}\left(\frac{\hbar \omega-2 \mu}{2 k_{B} T}\right)-i \frac{1}{\pi} \ln \left(\frac{\hbar \omega+2 \mu}{\hbar \omega-2 \mu}\right)\right],
\end{gathered}
$$

where $e$ is the elementary charge, $k_{B}$ is the Boltzmann constant, $\tau \approx 1$ ps is the relaxation time of carriers, $\hbar$ is the Dirac constant, $T$ is the temperature, $i$ is the imaginary unit.

At the same time, the surface conductivity of the graphene monolayer is defined by the sum of the intraband and the interband transitions:

$$
\hat{\sigma}=\hat{\sigma}_{\text {intra }}+\hat{\sigma}_{\text {inter }} \text {. }
$$

The value of the chemical potential under the influence of optical pumping with the frequency $\nu_{\text {pump }}=\Omega / 2 \pi$ and the energy which is more than $k_{B} T$, is defined as [15]:

$$
\mu=\hbar \nu_{F} \sqrt{\frac{2 \pi \alpha \tau_{R} I_{\Omega}}{\hbar \Omega}},
$$

where $\nu_{F}=106 \mathrm{~m} / \mathrm{s}$ is the Fermi velocity of charge carriers in graphene, $\alpha=1 / 137$ is the fine structure constant, $\tau_{R} \approx 1 \mathrm{~ns}$ is the time of a recombination of the electron-hole pairs, $I_{\Omega}$ is the graphene pumping intensity.

The dependences of the normalized surface conduction of graphene on the frequency $\omega$ with or without optical pumping and a wavelength of $1.5 \mu \mathrm{m}$ and intensity $I_{\Omega}=3 \mathrm{~W} / \mathrm{mm}^{2}$, which are calculated using the expressions (1) (4) are shown in Fig. 1. The tuning of graphene's chemical potential under the influence of optical pumping was shown to change the real and imaginary parts of the conductivity.

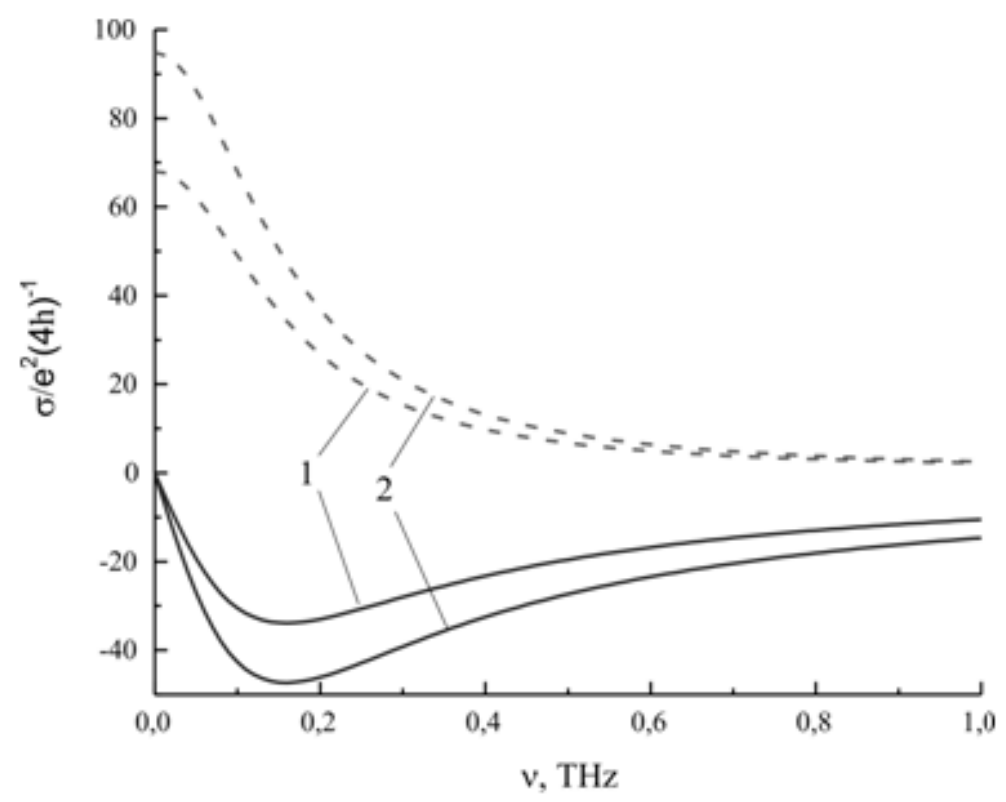

FIG. 1. The dispersion of the real and imaginary parts of the normalized conductivity of graphene at a temperature $T=293 \mathrm{~K}: 1$ - without pumping, 2 - under the optical pumping with wavelength of $1.5 \mu \mathrm{m}$ and intensity $I_{\Omega}=3 \mathrm{~W} / \mathrm{mm}^{2}$ (the solid lines represents the imaginary part of the conductivity, dashed lines - real part of conductivity) 
The real part of surface conductivity is known to be responsible for the absorption in the material, while the imaginary part of the surface conductivity allows one to calculate the phase difference between the electric field in the material and the excitation field, i.e., the delay in propagating through the material of the electromagnetic field. Information about the phase delay has a significant importance in the design of composite structures based on graphene and metamaterial, as the addition of graphene into the structure of metamaterial-based subwavelength resonator significantly shifts its resonant frequency.

As can be seen from Fig. 1, the largest difference between the imaginary part of surface conductivity with and without pumping is observed at a frequency of $0.18 \mathrm{THz}$, however, at that same frequency, significant losses are present. For this reason, the resonant frequency of the developed metamaterial was chosen in the higher frequency region of the spectrum, where the losses are minimal and the difference between the value $\operatorname{Im}(\sigma)$ with and without pumping is still significant. Frequencies near $0.4 \mathrm{THz}$ satisfy this condition. In addition, there are several radiation sources which operate at a frequency close to $0.4 \mathrm{THz}$ [16], that can be useful for experimental verification of the calculations.

\section{Optically tunable bandpass filter and method of calculation}

In this work, we studied hybrid structure based on the cross-like metasurface and optically pumped graphene in the terahertz frequency range. To describe all the effects occurring in such a composite structure, the usage of analytical methods is difficult, therefore, to address these issues, we applied a numerical approach.

Simulation of the electromagnetic fields in composite structure graphene/metamaterial was performed using the CST Microwave Studio software package with the frequency domain kernel which solves Maxwell's equations by the finite difference method. The boundary conditions were chosen along the $x$ and $y$ unit cell directions with the opened Floquet ports along the $z$ axis. As a result of this simulation, it is possible to obtain $S$-parameters ( $S_{11}$ is the amplitude of the reflection coefficient, $S_{12}$ is the amplitude of the transmission coefficient).

The graphene monolayer was defined as an infinitely thin sheet with surface impedance $Z=1 / \sigma$, where the conductivity $\sigma$ is described by the equations (1) - (4).

The filter is an artificial resonant structure made of an aluminum film with a thickness of $0.5 \mu \mathrm{m}$ having crosslike slots. This film is covered by a graphene monolayer and located on the dielectric substrate with thickness of $65 \mu \mathrm{m}$ and permittivity $\varepsilon=3$. The frequency spectrum of such a metasurface is determined by the width $K$ and the length $L$ of the cross-like slots, and also by the size $G$ of the unit cell. This form of a slot provides the polarization independence of the structure relative to the incident radiation. For numerical modeling, geometric parameters of the cross-like metasurface were selected such that the resonant frequency of the composite structure was about $0.4 \mathrm{THz}$, and the $Q$-factor was no less than 1.5 with a transmission coefficient value of $45 \%$ without optical pumping. The schematic of the proposed filter is presented in Fig. 2.

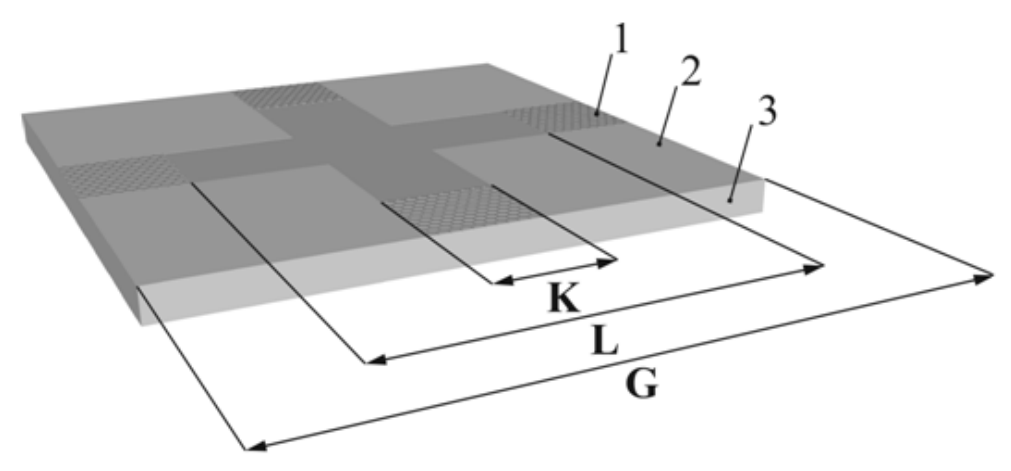

FIG. 2. Schematic of the elementary cell of the filter: 1 - graphene, 2 - aluminum, 3 - PET substrate

As a result of the simulation of the cross-like metasurface without graphene, we identified areas of the structure that make the largest contribution to the localization of the electromagnetic field. In accordance with this, we propose the structural unit cell, in which graphene covers the sections of the aluminum film, which are connect the end faces of the cross-like slots, as the electric field in these regions is maximized. 


\section{Results and discussion}

By optimizing the geometrical parameters of the cross-like resonator ( $K=45 \mu \mathrm{m}, L=180 \mu \mathrm{m}, G=440 \mu \mathrm{m})$, we have obtained the highest value for the quality factor $Q=1.9$ with the amplitude of the transmission coefficient of $45 \%$.

The dependence of the transmission coefficient amplitude on frequency for various pumping intensity values is shown in Fig. 3.

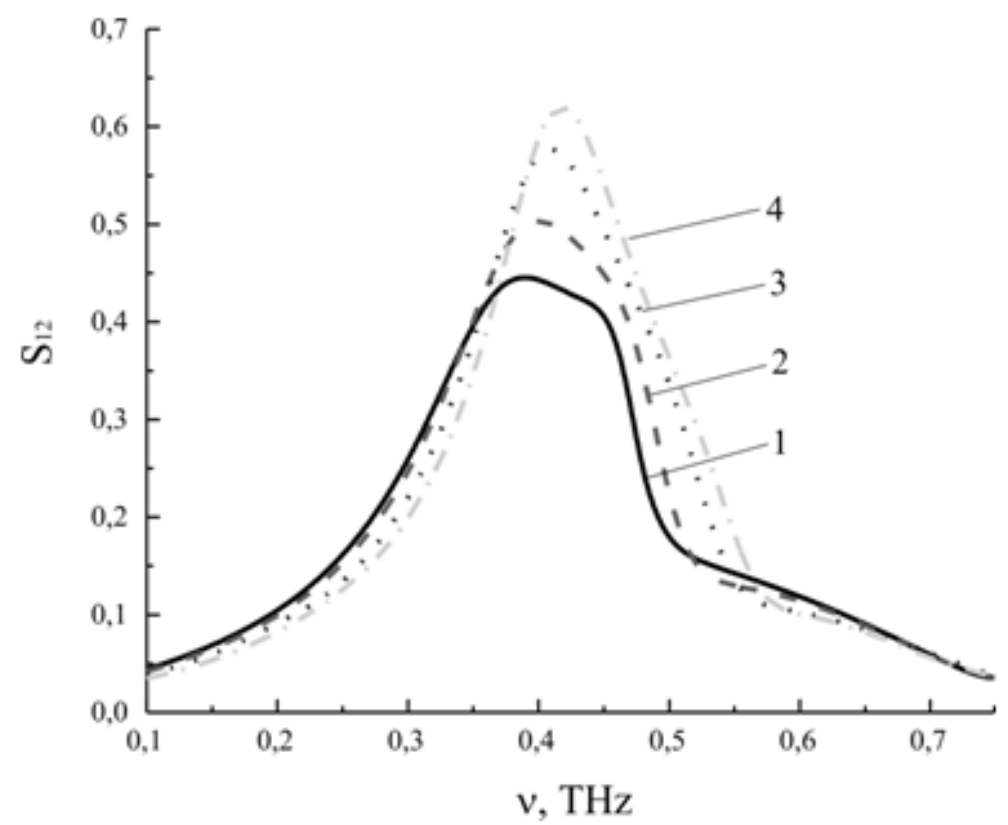

FIG. 3. The frequency dependence of the transmission coefficient amplitude for several pumping intensity values $\left(1-0 \mathrm{~W} / \mathrm{mm}^{2}, 2-2 \mathrm{~W} / \mathrm{mm}^{2}, 3-6 \mathrm{~W} / \mathrm{mm}^{2}, 4-10 \mathrm{~W} / \mathrm{mm}^{2}\right)$

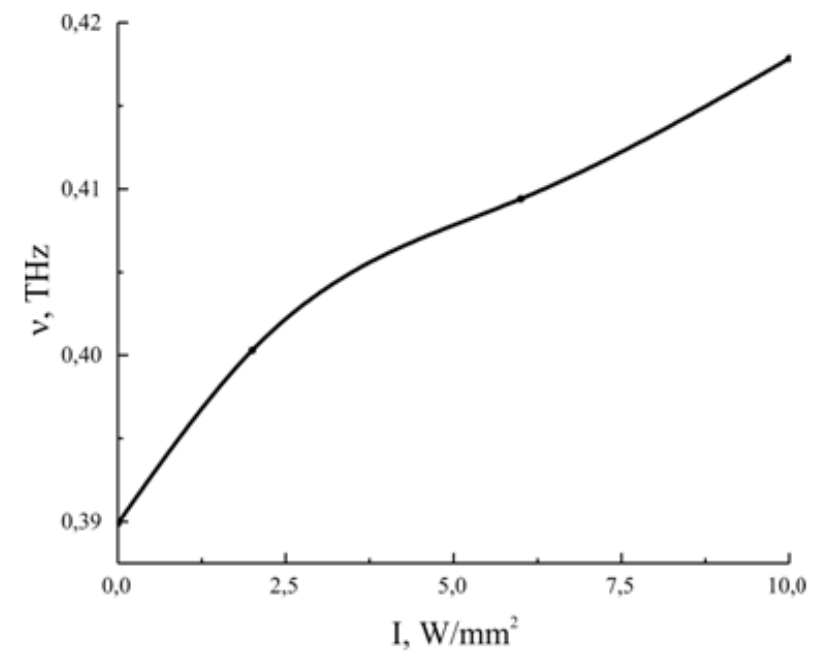

FIG. 4. The dependence of the central resonance frequency position on the pumping intensity

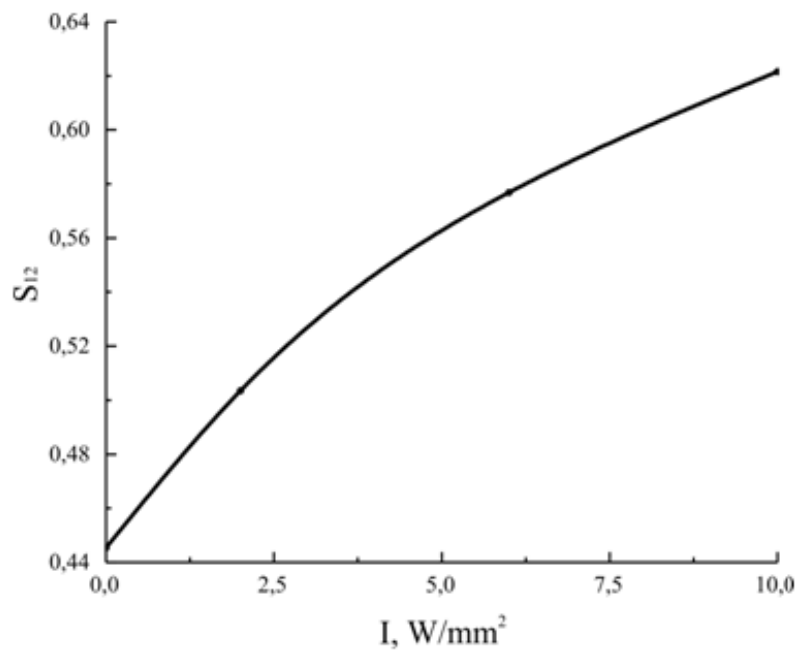

FIG. 5. The dependence of the transmission coefficient value on the pumping intensity

Figure 3 shows the frequency dependence for the amplitude of the transmission coefficient of the transmitted wave and its relative change for different graphene conductivity values, which is changed when graphene is pumped by optical radiation with a wavelength of $1.5 \mu \mathrm{m}$. The minimum value for the transmission coefficient is observed in the absence of optical pumping. Under optical pumping of the composite structure, the amplitude of the wave 
passing through the filter gradually increases; additionally, the $Q$-factor also increases, while and the resonant frequency is shifted to the higher frequency region. Figs. 4 and 5 show the dependences of the central resonant frequency and amplitude of the transmission coefficient, respectively, on the pumping intensity.

Thus, by means of numerical simulations, we have demonstrated the possibility for optical control of a $\mathrm{THz}$ wave passing through the hybrid filter based on the metamaterial and graphene, as the conductivity of graphene can be controlled by optical radiation. At the same time, the proposed filter is polarization-invariant due to structural symmetry.

\section{Acknowledgements}

The work was done with the support of the Government of the Russian Federation (Grant 074 - U01).

\section{References}

[1] Jepsen P.U., Cooke D.G., Koch M. Terahertz spectroscopy and imaging - Modern techniques and applications. Laser \& Photonics Reviews, 2011, 5 (1), P. 124-166.

[2] Adam A.J.L. Review of near-field terahertz measurement methods and their applications. Journal of Infrared, Millimeter, and Terahertz Waves, 2011, 32 (8-9), P. 976-1019.

[3] Song H.J., Nagatsuma T. Present and future of terahertz communications. IEEE Transactions on Terahertz Science and Technology, 2011, 1 (1), P. 256-263.

[4] Kim K.S., Zhao Y., et al. Large-scale pattern growth of graphene films for stretchable transparent electrodes. Nature, 2009 , 457 (7230), P. 706-710.

[5] Bonaccorso F., Sun Z., Hasan T., Ferrari A.C. Graphene photonics and optoelectronics. Nature photonics, 2010,4 (9), P. 611-622.

[6] Rana F. Graphene terahertz plasmon oscillators. IEEE Transactions on Nanotechnology, 2008, 7 (1), P. 91-99.

[7] He X.J., Li T.Y., et al. Electrically tunable terahertz wave modulator based on complementary metamaterial and graphene. Journal of Applied Physics, 2014, 115 (17), 17B903.

[8] Yang K., Liu S., et al. Graphene-based tunable metamaterial terahertz filters. Applied Physics Letters, 2014,105 (9), 093105.

[9] Gao W., Shu J., et al. High-contrast terahertz wave modulation by gated graphene enhanced by extraordinary transmission through ring apertures. Nano letters, 2014, 14 (3), P. 1242-1248.

[10] Lin Y.S., Qian Y., et al. Development of stress-induced curved actuators for a tunable THz filter based on double split-ring resonators. Applied Physics Letters, 2013, 102 (11), 111908.

[11] Lee S.H., Choi M., et al. Switching terahertz waves with gate-controlled active graphene metamaterials. Nature materials, 2012 , 11 (11), P. 936-941.

[12] Weis P., Garcia-Pomar J.L., et al. Spectrally wide-band terahertz wave modulator based on optically tuned graphene. ACS nano, 2012, 6 (10), P. 9118-9124.

[13] Ren L., Zhang Q., et al. Terahertz and infrared spectroscopy of gated large-area graphene. Nano Lett., 2012, 12, P. $3711-3715$.

[14] Falkovsky L.A. Optical properties of graphene. J. Phys. Conf. Ser., 2008, 129, 012004.

[15] Ryzhii V., Ryzhii M., Otsuji T. Negative dynamic conductivity of graphene with optical pumping. J. Appl. Phys., $2007,101,083114$.

[16] Lewis R.A. A review of terahertz sources. J. Appl. Phys. D: Applied Physics, 2014, 47 (37), 374001. 